\title{
Impact on long-term respiratory and neurologic sequelae of Sars-Cov- 2 in the first year of global pandemic era: A mini review
}

\author{
Weimer LE ${ }^{1 *}$, Cattari G ${ }^{2}$, Binelli A ${ }^{1}$, Fanales-Belasio $E^{3}$, Poddighe AF ${ }^{2}$ and Sensi $F^{2}$ \\ ${ }^{1}$ National Center for Global Health, Istituto Superiore di Sanità, Rome, Italy \\ ${ }^{2}$ SSD Lungodegenza-ASSL Sassari-ATS Sardegna, Italy \\ ${ }^{3}$ Department of Infectious Diseases, MIPI, Istituto Superiore di Sanità, Rome, Italy
}

\begin{abstract}
The world has been the stage for a new coronavirus, officially named severe acute respiratory syndrome coronavirus 2 (SARS-CoV-2), causing the coronavirus disease 2019 (COVID-19).

The result of the rapid and uncontrolled epidemic, countries healthcare systems have shown different reactions in surveillance, diagnosis, and treatment. In the first year of global pandemic, more than 116 million cases have been confirmed by the World Health Organization, with more than 2.6 million deaths registered worldwide. While clinical trials for safe and effective antiviral agents are ongoing, and vaccine development programs are being accelerated, long-term sequelae of SARS-CoV-2 infection have become increasingly recognized and concerning. Although the upper and lower respiratory tracts are the main sites of entry of SARS-CoV-2 into the body, resulting in COVID-19 pneumonia as the most common presentation, acute lung damage may be followed by pulmonary fibrosis and chronic impairment of lung function, with impaired quality of life. Also, coronaviruses have neuroinvasive and neurotropic properties and cause symptoms of fatigue and muscle ache, accompanied by anxiety, depression, pain, and sleep difficulties and severe neurological complications such as encephalitis and Guillain-Barré.

The purpose of the present review is to summarize our understanding of neurological and respiratory disorders associated with COVID-19, bringing current evidence of the potential mechanisms of injury (immune mediated, direct viral damage, hypoxia and hypercoagulability).

These aspects are relevant for correct diagnosis, treatment and follow-up post-Covid for prevention of the potential risk of pulmonary fibrosis and severe neurological sequelae.
\end{abstract}

\section{Introduction}

The world has been the stage for a new coronavirus, officially named severe acute respiratory syndrome coronavirus 2 (SARS-CoV-2), causing the coronavirus disease 2019 (COVID-19).

The result of the rapid and uncontrolled epidemic, countries healthcare systems have shown different reactions in surveillance, diagnosis, and treatment. In the first year of global pandemic, more than 116 million cases have been confirmed by the World Health Organization, with more than 2.6 million deaths registered worldwide [1].

While clinical trials for safe and effective antiviral agents are ongoing, and vaccine development programs are being accelerated, long-term sequelae of SARS-CoV-2 infection have become increasingly recognized and concerning. Although the upper and lower respiratory tracts are the main sites of entry of SARS-CoV-2 into the body, resulting in COVID-19 pneumonia as the most common presentation, acute lung damage may be followed by pulmonary fibrosis and chronic impairment of lung function, with impaired quality of life. Also, coronaviruses have neuroinvasive and neurotropic properties and cause symptoms of fatigue and muscle ache, accompanied by anxiety, depression, pain, and sleep difficulties and severe neurological complications such as encephalitis and Guillain-Barré.
The purpose of the present review is to summarize our understanding of neurological and respiratory disorders associated with COVID-19, bringing current evidence of the potential mechanisms of injury (immune mediated, direct viral damage, hypoxia and hypercoagulability).

These aspects are relevant for correct diagnosis, treatment and follow-up post-Covid for prevention of the potential risk of pulmonary fibrosis and severe neurological sequelae.

\section{Pulmonary complications}

While the COVID-19 pandemic affecting many systems, the most affected one is the respiratory system, and the cases that we encounter mostly occur with viral pneumonia with fibrosis.

${ }^{\star}$ Correspondence to: Weimer LE, National Center for Global Health, Istituto Superiore di Sanità, Rome, Italy, E-mail: liliana.weimer@iss.it

Key words: crespiratory and neurological sequelae, Sars-Cov-2

Received: March 09, 2021; Accepted: March 15, 2021; Published: March 18, 2021 
Pulmonary fibrosis can develop either following chronic inflammation or as a primary, genetically influenced, and age-related fibroproliferative process. World Health Organization (WHO) estimates that $80 \%$ of the cases are asymptomatic or mild, $20 \%$ of them vary in severity with clinical conditions such as respiratory failure, hyperinflammatory response, thromboembolism and acute respiratory distress syndrome. The long-term sequalae of Sars-COV-2, it is predicted that respiratory systems are more probability of injury (immune mediated, direct viral damage, hypoxia and hypercoagulability) similar to SARS and middle east respiratory syndrome (MERS). In a study investigating the effects of SARS disease, people who had the disease were examined for one year, and it was found that $23 \%$ of these patients had impaired lung functions and decreased exercise capacity [2].

\section{Emerging clinical evidence of pulmonary fibrosis following SARS-CoV-2 infection}

Acute lung injury, attempt at repair by fibroproliferation, and lung remodeling occur in COVID-19 disease, much like it does in other coronavirus infections. This leads to a potential increase in the risk of pulmonary fibrosis occurring as a sequela of COVID-19. Fibrotic changes have been found on chest CT scans in COVID-19 patients. In a study of 62 patients by Zhou, et al. fibrotic changes were seen in 21 (33.9\%) patients, with this finding more likely to occur in advancedphase disease (8-14 days after the onset of symptoms) than early phase of the disease ( $\leq 7$ days after the onset of symptoms) [3]. Similarly, Pan, et al. reported fibrotic changes on the chest CT scan of 11 out of 63 patients taken during the acute illness [4]. These imaging findings are supported by autopsy reports. Reports on 4 patients who died of COVID-19 pneumonia reveal features of diffuse alveolar damage with areas of consolidation by fibroblastic proliferation and deposition of ECM and fibrin in the alveolar spaces [5]. Similarly, lung explants from 3 patients who had lung transplant for end-stage ARDS show extensive fibrosis of the lungs [6]. The finding of fibrotic changes early in the disease suggests an attempt at repair following pulmonary injury. It is however too early in the process of the disease to determine if this finding would resolve with time or progress to fixed pulmonary fibrosis [7].

\section{Strategies for reducing the risk of SARS-CoV-2 infection- associated pulmonary fibrosis}

Pulmonary fibrosis is associated with a significant reduction in the quality of life [8]. Two disease-modifying drugs, nintedanib and pirfenidone, have shown promise in clinical trials in slowing down the decline in pulmonary function. Nintedanib is a tyrosine kinase inhibitor active against growth factor receptors with intrinsic tyrosine kinase activity such as EGFR, VEGFR, and PDGFR. The exact mechanism of action of pirfenidone is not known; however, it is thought to have antiinflammatory, antioxidant, and antifibrotic properties. Neither of these two drugs has demonstrated significant improvement in symptoms or long-term survival benefit. Lung transplantation is the only treatment option with demonstrable increased survival in selected patients with IPF. However, only a few receive this intervention. Chen, et al. reported 3 critically ill patients with COVID-19-related pulmonary fibrosis who had lung transplantation. Two of the patients survived the procedure [6]. Considering the challenges of treating pulmonary fibrosis, there should be greater focus on strategies aimed at reducing the risk among COVID-19 patients. These strategies should be targeted at limiting the factors that perpetuate the cycle of lung injury, inflammatory response, and fibroproliferation.
The viral protease inhibitors: lopinavir/ritonavir, with the RNA polymerase inhibitors: favipiravir and remdesivir, are antiviral agents currently used in many clinical trials in a bid to find an effective therapy against SARS-CoV-2 [8].

Other medications with antiviral and immunomodulatoryproperties being considered include nitazoxanide and ivermectin. Although these medications have shown in vitro activity against SARS-CoV-2, further studies are required to determine their efficacy in the treatment of COVID-19. With cytokine storm due to hyperinflammation being a major factor implicated in lung damage in SARS-CoV-2 infection, the use of immunosuppressive agents has been widely recommended in the treatment of Sars-Cov-2. Immunosuppressive agents that are currently under consideration include the IL-1 receptor blocker anakinra which has demonstrated improved survival benefits in cytokine storm due to sepsis and IL-6 receptor blocker. Tocilizumab is currently registered for a multicenter clinical trial in COVID-19 patients. Despite the common use of corticosteroids, there is little evidence supporting its clinical benefit. The use of convalescent plasma has shown some promise in clinical trials. In addition, mesenchymal stem cells, having multipotent capacity to replace damaged alveolar epithelium, secrete antiinflammatory factors as well as inhibit fibroproliferation and have been considered in the treatment of ARDS, the leading cause of mortality in COVID-19 disease. The use of mesenchymal stem cells is currently undergoing clinical trials.

As the search for an effective therapy against COVID-19 continues, attention should equally be focused on other modifiable factors that could increase the risk of pulmonary fibrosis. The risk of ventilatorinduced lung injury should be minimized by the use of protective lung ventilation settings with low tidal volumes and low inspiratory pressures. This has been shown to reduce the relative risk of mortality in ARDS by $30 \%$ [9]. In addition, continuous lung injury has been considered a major factor in the development of lung fibrosis. Therefore, patients should be educated prior to discharge on limiting exposure to environmental factors associated with increased lung injury. Smoking cessation has been shown to slow the accelerated pulmonary function decline in chronic obstructive pulmonary disease, suggesting a reduction in pulmonary inflammatory and remodeling process. In measures to reduce particulate inhalation from indoor and outdoor air pollution, an implicated factor in the development of pulmonary fibrosis should be considered. In addition, convalescent patients should be followed up with pulmonary function tests and high-resolution CT scans to monitor changes in lung architecture and function.

\section{Neurological features of SARS-COV-2 infection}

Patients in critical condition were more likely to develop neurological manifestations than those with mild or moderate presentations of the disease (45.5\% vs. $30.2 \%)$ [10]. Neurological involvement was classified according to localization: central nervous system (CNS), peripheral nervous system (PNS), and musculoskeletal. Among the CNS manifestations which occurred in $24.8 \%$ of patients, the most common were dizziness $(16.8 \%)$, headache $(13.1 \%)$, altered level of consciousness (7.5\%), and acute cerebrovascular disease (2.8\%), defined as ischemic or hemorrhagic stroke. Among the PNS manifestations, which presented in $8.9 \%$ of patients, the most prevalent were hypogeusia (5.6\%), hyposmia (5.1\%), and neuralgia $(2.3 \%)$. Musculoskeletal involvement, defined as myalgia (muscle pain) and elevated levels of creatine kinase in the blood (>200 U/L) were found in $10.7 \%$ of patients. In addition to this, critically ill patients had disorders of multiple organs, including the liver, kidneys, and muscular damage. 
An Italian study showed that three out of five cases were consistent with an axonal variant of Guillain-Barré syndrome, and with a demyelinating process in two others\%) [11]. The diagnosis was based on positive test results of nasopharyngeal samples for SARS-CoV-2 at the onset of the syndrome in four patients, and one had reactive serologic test for the virus. There was an interval of five to ten days between the onset of COVID-19 and the first symptoms. Lower-limb weakness and paresthesia were found in four patients, and facial diplegia, ataxia, and paresthesia in another. All the CSF samples showed negative results on RT-PCR assay for SARS-CoV-2, with normal white blood cell counts (fewer than 5 leukocytes $/ \mathrm{mm}^{3}$ ). Three patients had elevated protein levels ( $>40 \mathrm{mg} / \mathrm{dL}$ ) in CSF. Electromyography showed fibrillation potentials in four patients (three in the early phase and one on the 12th day). The mechanisms underlying cerebrovascular disease manifestations in COVID-19 are probably multifactorial (viral, immune, hypoxia, and hypercoagulability). For this reason, there are reports of acute stroke associated with COVID-19 not only in individuals with risk factors, but also in the younger population. Table 1 summarizes the spectrum of neurological manifestations associated with Sars-Cov-2, published by Puccioni -Sohler M, et al. [12].

\section{Laboratorial diagnosis}

Available laboratory tests capable of detecting SARS-CoV-2 are based on molecular biology principles (real-time reverse transcription polymerase chain reaction [RT-PCR]) and immunological testing (specific IgG and IgM antibodies). Viral detection using real-time RT-PCR in respiratory samples from the oropharynx, nasopharynx, sputum, and/or endotracheal aspirate or bronchoalveolar lavage in severe respiratory disease continues to be the preferred method of laboratory testing for the diagnosis of symptomatic patients in the acute phase of the disease (the first week after onset of symptoms). A negative RT-PCR assay result does not exclude the infection, considering that the sensitivity of RT-PCR is approximately $60 \%$. Moreover, the viral load may be undetectable during the mean incubation period (first five days). After the second week of infectious symptoms of the disease, when patients develop an immune response, immunological tests using blood samples (validated serological tests) are recommended. Neurological

Table 1. Neurologic manifestations of Sars-Cov-2

\begin{tabular}{|l|}
\hline Central nervous system diseases \\
\hline Encephalitis, meningitis, myelitis, meningoencephalitis \\
\hline CNS demyelinating disease \\
\hline Post-infectious acute disseminated encephalomyelitis \\
\hline Post-infectious brainstem encephalitis \\
\hline Encephalopathy, Delirium \\
\hline Movement disorders \\
\hline Acute hemorrhagic necrotizing encephalopathy \\
\hline $\begin{array}{l}\text { Cerebrovascular disease: Ischemic and haemorrhagic stroke, cerebral venous sinus } \\
\text { thrombosis, venous and arterial thromboses, subarachnoid haemorrhage associated with } \\
\text { immune thrombocytopenic purpura }\end{array}$ \\
\hline Peripheral nervous system \\
\hline Guillain-Barré syndrome \\
\hline Miller Fisher syndrome \\
\hline Mononeuropathy \\
\hline Polyneuritis cranialis \\
\hline Optic neuritis \\
\hline Dysautonomia \\
\hline Muscle involvement \\
\hline Myalgia \\
\hline Myopathies \\
\hline Rhabdomyolysis \\
\hline
\end{tabular}

disorders caused by SARS-CoV-2 have been mainly reported during the first two weeks after the onset of infectious symptoms. So far, few cases of encephalitis/meningitis with positive RT-PCR assays of the CSF have been described. This can be attributed to several reasons: the RTPCR assay of CSF may yield negative results when performed early; the method has limited sensitivity associated with false negative tests in the CSF; and CNS viral clearance in cases of early neuroinvasion induces a low viral load in the CSF. On the other hand, not all neurological complications of COVID-19 occur due to direct viral action. Systemic viral infection may be the trigger for neurological autoimmunemediated diseases. Post-infectious and parainfectious nervous system autoimmune diseases, as well as metabolic and cerebrovascular disorders are reported to be associated with COVID-19. Under these conditions, the virus may not be detected in the CSF. Therefore, the laboratory results should be evaluated along with the nervous system condition, including immunological and molecular examinations of all samples that detect the presence of the virus in the patient, not only in CSF. Specific IgM in CSF has been found in cases of negative RTPCR assays. This marker may be promising for the diagnosis of neuroCOVID-19.

In addition to analyzing the presence of the virus in the CSF, the inflammatory profile (pleocytosis, high level of protein, blood-CSF barrier dysfunction, and intrathecal synthesis of immunoglobulins) and albuminocytological dissociation (normal cell count and high levels of protein) should be investigated in CSF. Although not every COVID-19 patient will test positive for the virus in the CSF exam, the appearance of neurological symptoms in the course of infection with SARS-CoV-2 indicates that it may still represent a neurologic manifestation associated with infection [12].

\section{Evidence of neuropathogenesis}

The mechanism through which the virus affects the nervous system is not entirely clear yet. Some authors have proposed hypotheses based on evidence related to the SARS-CoV-2 and other coronaviruses, particularly SARS-CoV and MERS-CoV. A possible mechanism is direct damage due to the presence of the virus in the CNS. This could occur by two main pathways. First, via the hematogenous route, which has already been described for other coronaviruses. SARS-CoV-2 particles have already been identified in capillary endothelia and neurons of frontal lobe brain sections in an autopsy of a COVID-19 patient, corroborating this hypothesis. Second, via the neuronal route, by which the virus infects nerve endings and migrates through the synapses until it reaches the CNS, which has already been described in SARS-CoV. The olfactory nerve is mainly held responsible for the infection of the CNS through the neuronal pathway due to its proximity to the nasal cavity, a common locus of virus infection, and the communication of the nasal epithelium with the olfactory bulb, through the cribriform plate of the ethmoid bone, thereby allowing the entrance of the virus. This possibility is supported by the findings of hyposmia or anosmia in the early stages of COVID-19. In addition, brain magnetic resonance imaging showed signal alteration in the olfactory bulbs and frontal lobe of a patient with anosmia with SARS-CoV-2 infection. After disappearance of the symptoms, the control image was also normal.

The functional receptor used by SARS-CoV-2 for cellular invasion is the angiotensin-converting enzyme 2 (ACE2) receptor, expressed in different tissues, including the endothelium of small blood vessels in the brain's microcirculation, and, to a lesser extent, in cells of the CNS (neurons and glia cells). The virus infects the cells using the S1 spike protein to interact with the cellular ACE2 receptors. This could explain 
how the virus overcomes the blood-brain (brain capillaries endothelial cells) and blood-CSF (choroid plexus epithelium of the ventricles) barriers to invade the CNS via the hematogenous route.

The hypotheses of direct infection of the CNS is also corroborated by the description of patients with COVID-19 from whom the virus was isolated in samples of CSF, which characterizes a condition of viral encephalitis, as well as the case of another patient with neck stiffness, seizures, and lowered consciousness level, who also had SARS-CoV-2 in the CSF, but not on the nasopharyngeal swab, which characterizes viral meningitis. In addition, the detection and sequence of the virus was demonstrated in the CSF of a patient suspected of clinically isolated syndrome, a CNS demyelinating disorder.

Other mechanisms that would account for neurological conditions in COVID-19 are hypoxia and immune injury. Hypoxia, caused by pulmonary infections, generates swelling in the brain and intracranial hypertension, impairing blood flow to the brain and leading to acute ischemic stroke. Immune injury, explained by the so-called proinflammatory "cytokine storm" provoked by SARS-CoV-2 infection, can cause damages to brain cells and to the skeletal muscle. Activated monocytes in the bloodstream produce proinflammatory cytokines that may damage the blood-brain or blood-CSF barriers, oligodendrocytes, neurons, and muscles.

Another mechanism that has been suggested is based on the fact that ACE2 participates in the regulatory mechanisms both of blood pressure and of blood flow to the brain, with reduced expression in patients previously suffering from high blood pressure. With SARS$\mathrm{CoV}-2$ infection, this function becomes even more impaired, leading to hypertensive peaks that may result in brain hemorrhage. In parallel, patients with COVID-19 present elevated levels of D-dimer and antiphospholipid antibody, which may be related to thrombotic phenomena and can increase the probability of cerebrovascular diseases. \%) [12].

\section{Conclusion}

SARS-CoV-2 has continued to spread across the world, infecting millions of individuals in the process. Like previous human coronavirus outbreaks, pulmonary fibrosis and neurological symptoms has been recognized as a potential sequela among survivors. Virus-induced lung injury, immune response, and attempts at healing are central to the process of fibrogenesis.
Predictors of pulmonary fibrosis putatively include advanced age, illness severity, smoking, and chronic alcoholism. With no proven effective targeted therapy against pulmonary fibrosis, risk reduction measures should be directed at limiting illness severity and protecting the lung from other incidental injuries.

Finally, since SARS-CoV-2 has neuroinvasive and neurotropic properties, early recognition can help initiate treatment and isolation early, to prevent clinical worsening and spread of the virus and the underreporting of neurological cases associated with COVID-19.

\section{References}

1. World Health Organization (WHO). Novel Coronavirus (2019-nCoV). Situation report 191. Geneva: WHO.

2. Hui DS, Wong KT, Ko FW, Tam LS, Chan DP, et al. (2005) The 1-year impact of severe acute respiratory syndrome on pulmonary function, exercise capacity, and quality of life in a cohort of survivors. Chest 128: 2247-2261. [Crossref]

3. Zhou S, Wang Y, Zhu T, Xia L (2020) CT features of coronavirus disease 2019 (COVID-19) pneumonia in 62 patients in Wuhan, China. Am J Roentgenol 214: 12871294. [Crossref]

4. Pan Y, Guan H, Zhou S, Wang Y, Li Q, et al. (2020) Initial CT findings and temporal changes in patients with the novel coronavirus pneumonia (2019-nCoV): A study of 63 patients in Wuhan, China. Eur Radiol 30: 3306-3309. [Crossref]

5. Tian S, Xiong Y, Liu H, Niu L, Guo J, et al. (2020) Pathological study of the 2019 novel coronavirus disease (COVID-19) through postmortem core biopsies. Mod Pathol 33: 1007-1014.

6. Chen JY, Qiao K, Liu F, Wu B, Xu X, et al. (2020) Lung transplantation as therapeutic option in acute respiratory distress syndrome for COVID-19-related pulmonary fibrosis. Chin Med J 133: 1390-1396. [Crossref]

7. Ye z, Zhang Y, Wang Y, Huang Z, Song B (2020) Chest CT manifestations of new coronavirus disease 2019 (COVID-19): A pictorial review. Eur Radiol 30: 4381-4389.

8. Ojo AS, Balogun SA, Williams OT, Ojo OS (2020) Pulmonary fibrosis in COVID-19 survivors: Predictive factors and risk reduction strategies. Pulm Med 2020: 6175964. [Crossref]

9. Hoegl S, Zwissler B (2017) Preventing ventilator-induced lung injury-what does the evidence say? J Thorac Dis 9: 2259-2263. [Crossref]

10. Mao L, Jin H, Wang M, Hu Y, Chen S, et al. (2020) Neurologic manifestations of hospitalized patients with coronavirus Disease 2019 in Wuhan, China. JAMA Neurol 77: 683-690. [Crossref]

11. Toscano G, Palmerini F, Ravaglia S, Ruiz L, Invernizzi P, et al. (2020) Guillain-barré syndrome associated with SARS-CoV-2. N Engl J Med 382: 2574-2576. [Crossref]

12. Puccioni-Sohler M, Poton AR, Franklin M, da Silva JS, Brindeiro R, et al. (2020) Current evidence of neurological features, diagnosis, and neuropathogenesis associated with COVID-19. Rev Soc Bras Med Trop 53.

Copyright: (C2021 Weimer LE. This is an open-access article distributed under the terms of the Creative Commons Attribution License, which permits unrestricted use, distribution, and reproduction in any medium, provided the original author and source are credited. 